\title{
Psychological interventions for trauma in individuals who have psychosis: a systematic review and meta-analysis
}

Article

Accepted Version

Sin, J. and Spain, D. (2017) Psychological interventions for trauma in individuals who have psychosis: a systematic review and meta-analysis. Psychosis, 9 (1). pp. 67-81. ISSN 17522439 doi: https://doi.org/10.1080/17522439.2016.1167946 Available at https://centaur.reading.ac.uk/71142/

It is advisable to refer to the publisher's version if you intend to cite from the work. See Guidance on citing.

To link to this article DOI: http://dx.doi.org/10.1080/17522439.2016.1167946

Publisher: Taylor \& Francis

All outputs in CentAUR are protected by Intellectual Property Rights law, including copyright law. Copyright and IPR is retained by the creators or other copyright holders. Terms and conditions for use of this material are defined in the End User Agreement.

www.reading.ac.uk/centaur 
Central Archive at the University of Reading

Reading's research outputs online 
Title of manuscript

Are psychological interventions effective for treating symptoms of trauma in individuals who have psychosis? - A systematic review and meta-analysis

Short/ running title: Psychological interventions for comorbid trauma \& psychosis

\title{
Authors' details
}

First and corresponding author: Jacqueline $\operatorname{Sin}^{1,2}$

Position and Affiliation: King's College London Prize Fellowship - NIHR BRC Clinical Lecturer

\author{
${ }^{1}$ Health Service \& Population Research Department \\ Institute of Psychiatry, Psychology \& Neuroscience \\ King's College London
}

Full address: David Goldberg Centre, de Crespigny Park, London SE5 8AF

Email address: Jacqueline.sin@kcl.ac.uk

Telephone number: 02078485421

${ }^{2}$ NIHR Post Doctoral Research Fellow

Population Health Research institute

St George's, University of London

\author{
Second and last author: Debbie Spain ${ }^{3}$ \\ Position and affiliation: NIHR Clinical Doctoral Research Fellow \\ ${ }^{3} \mathrm{MRC}$ Social, Genetic and Developmental Psychiatry Centre \\ Institute of Psychiatry, Psychology \& Neuroscience \\ King's College London \\ Email address: Debbie.spain@kcl.ac.uk
}

\begin{abstract}
Background

Psychological interventions, in particular those derived from cognitive-behavioural therapy (CBT) frameworks and Eye Movement Desensitisation and Reprocessing (EMDR), are effective for reducing posttraumatic stress disorder (PTSD) symptoms and associated distress. However studies to date have tended to exclude people who have psychosis; a clinical population who are known to be at risk of experiencing trauma. Whether people with psychosis also benefit from trauma-focused psychological therapies (TFPT) warrants further investigation.
\end{abstract}

\section{Method}

A systematic search for randomised controlled trials (RCTs) was undertaken. Data were synthesised using narrative and meta-analytical approaches.

\section{Results}

Five studies met the review inclusion criteria. Study findings overall, indicate that TFPI are clinically effective for reducing intrusive thoughts and images, negative thoughts and beliefs 
Psychological interventions for comorbid trauma \& psychosis

associated with traumatic memories, hypervigilance, and avoidance. Limited data were available about the utility of interventions for improving mood, anxiety and quality of life. Attrition rates were comparable for participants offered active and control conditions; the number of adverse events reported was low.

\section{Conclusion}

Findings are consistent with those reported for non-psychosis populations. Further studies should establish which intervention modalities glean more favourable outcomes, and are more acceptable for this clinical population.

\section{Keywords}

\section{psychosis | post-traumatic stress disorder | PTSD | trauma | psychological interventions | EMDR | systematic review}

\section{Acknowledgements}

The authors acknowledge the expert input provided by the Cochrane Schizophrenia Group (CSG) Trials Search Coordinator, Farhad Shokraneh who devised and executed the search strategies for trials in the Cochrane Schizophrenia Group's Study-Based Register of Controlled Trials.

The current review is derived from a Cochrane systematic review entitled "Psychological interventions for PTSD in people with severe mental illness" but has a broader remit. We thank the co-authors Marie Furuta, Trevor Murrells, Ian Norman, and the CSG editorial team members for their contributions to the Cochrane systematic review.

JS is supported by the National Institute for Health Research (NIHR) Post Doctoral Research Fellowship (Reference: PDF-2015-08-035, 2016-2020) and also in part by the NIHR Biomedical Research Centre based at Guy's and St Thomas' NHS Foundation Trust and King's College London (2015 - 2017). DS is funded by a NIHR Clinical Doctoral Research Fellowship (Reference: CDRF-03-059, 2013-2016). The views expressed are those of the authors and not necessarily those of the NHS, the NIHR or the Department of Health.

\section{Declaration of interest}

Both authors declared no conflict of interests. JS was involved in the "Cognitive behaviour therapy for post-traumatic stress disorder and schizophrenia (ISRCTN67096137)" (Steel, 2010) in the capacity of a trial therapist from 2009 to 2010.

Both authors have contributed to the writing of the paper and approved this manuscript. 
Psychological interventions for comorbid trauma \& psychosis

Title

Are psychological interventions effective for treating symptoms of trauma in individuals who have psychosis? - A systematic review and meta-analysis

\section{Abstract}

\section{Background}

Psychological interventions, in particular those derived from cognitive-behavioural therapy (CBT) frameworks and Eye Movement Desensitisation and Reprocessing (EMDR), are effective for reducing posttraumatic stress disorder (PTSD) symptoms and associated distress. However studies to date have tended to exclude people who have psychosis; a clinical population who are known to be at risk of experiencing trauma. Whether people with psychosis also benefit from trauma-focused psychological therapies (TFPT) warrants further investigation.

\section{Method}

A systematic search for randomised controlled trials (RCTs) was undertaken. Data were synthesised using narrative and meta-analytical approaches.

\section{Results}

Five studies met the review inclusion criteria. Study findings overall, indicate that TFPI are clinically effective for reducing intrusive thoughts and images, negative thoughts and beliefs associated with traumatic memories, hypervigilance, and avoidance. Limited data were available about the utility of interventions for improving mood, anxiety and quality of life. Attrition rates were comparable for participants offered active and control conditions; the number of adverse events reported was low.

\section{Conclusion}

Findings are consistent with those reported for non-psychosis populations. Further studies should establish which intervention modalities glean more favourable outcomes, and are more acceptable for this clinical population.

Keywords: psychosis | post-traumatic stress disorder | PTSD | trauma | psychological interventions | EMDR | systematic review 
Psychological interventions for comorbid trauma \& psychosis 
Psychological interventions for comorbid trauma \& psychosis

\section{Introduction}

Post-traumatic stress disorder (PTSD) is a mental health condition which can develop as a result of witnessing, or experiencing, single or multiple traumatic events, incurring a perceived threat to life or significant risk to physical well-being, and intense fear, horror, or helplessness (APA, 2013). DSM-5 (APA, 2013) outlines four distinct symptom clusters (one more than described in DSM-IV-Tr, APA, 2000), as follows: re-experiencing (for example intrusive thoughts/ images related to the trauma); avoidance (for example, sites or cues associated with the traumatic event); arousal or hypervigilance (for example, 'fight or flight' responses, or panic symptoms); and negative thoughts and beliefs.

PTSD prevalence estimates are reported to fall between $0.4 \%$ and 3.5\% (Bisson, Roberts, Andrew, Cooper, \& Lewis, 2013; Darves-Bornoz et al., 2008; Kessler, Sonnega, Bromet, Hughes, \& Nelson, 1995; NICE, 2005). Some populations, however, are at increased risk of experiencing trauma and adversity, in particular, people who have psychosis (Bebbington et al., 2004; Fisher et al., 2013; Morrison, Frame, \& Larkin, 2003; Read et al., 2008): PTSD prevalence rates are approximately $30 \%$, including individuals meeting full-blown PTSD diagnostic criteria, or sub-threshold diagnostic presentations (Brunet, Birchwood, Upthegrove, Michail, \& Ross, 2012; Kilcommons \& Morrison, 2005; NICE, 2014). Data from cross-sectional and longitudinal studies indicate that some social factors may predispose individuals to developing both psychosis and PTSD (Read et al., 2008). These include traumatic events occurring during childhood, such as abuse, or sustained bullying (Bebbington et al., 2011; Bebbington et al., 2004; Cunningham, Hoy, \& Shannon, 2015; Varese et al., 2012); and adulthood, such as vulnerability to exploitation and victimisation (Darves-Bornoz et al., 2008; Mueser, Lu, Rosenberg, \& Wolfe, 2010). Additionally, several studies have concluded that positive psychotic symptoms, such as persecutory delusions, can also understandably be perceived as traumatic (Jackson et al., 2009; Kilcommons \& Morrison, 2005). The combination of psychotic symptoms and PTSD likely results in an exacerbation in low mood and anxiety, functional impairment, and reduced quality of life (Mueser, Rosenberg, \& Rosenberg, 2009; Read et al., 2008).

UK and Australian Clinical Guidelines pertaining to adults experiencing single event trauma (ACPMH, 2013; NICE, 2005, 2013) recommend a course of eight to 12 individual outpatient sessions of trauma-focused cognitive behavioural therapy (TFCBT including prolonged exposure), and/or eye movement desensitisation and reprocessing (EMDR). There are some commonalities between TFCBT and EMDR, which are collectively referred to as 'trauma- 
Psychological interventions for comorbid trauma \& psychosis

focused psychological therapies' (TFPT) (Bisson et al., 2013; Schnyder et al., 2015): both treatments encourage individuals to make sense of, and process traumatic memories, beliefs and attributions about traumatic events, and their impact; and develop more effective strategies for ameliorating symptoms.

To date, empirical research investigating effectiveness and acceptability of TFPT have tended to exclude individuals with a concurrent diagnosis of psychosis (Mueser et al., 2010; NICE, 2014); which mirrors health inequalities evident in clinical settings (The Schizophrenia Commission, 2012). This may be due to:1) diagnostic overshadowing rendering it difficult to disentangle symptoms between disorders (Calvert, Larkin, \& Jellicoe-Jones, 2008; Jones \& Steel, 2014); 2) concerns that individuals may find it hard to engage in psychological therapies (Callcott, Standart, \& Turkington, 2004; Gairns, Alvarez-Jimenez, Hulbert, McGorry, \& Bendall, 2015); 3) worry that interventions may exacerbate positive psychotic symptoms (Gairns et al., 2015; Kilcommons \& Morrison, 2005); and 4) apprehension that individuals may disengage mid-way during a course of treatment. Hence, relatively little is known about the effectiveness of TFPT for individuals with psychosis, although considerable evidence indicates that CBT and psychosocial interventions can reduce psychotic symptoms, distress, and co-morbidities, such as depression and anxiety (Birchwood, 2003; Garety et al., 2008; NICE, 2014; Turkington, Kingdon, \& Turner, 2002). Importantly, rates of adverse effects are not increased for this group (NICE, 2014).

This review had three aims: 1) to synthesise evidence about the effectiveness of TFPT for individuals with psychosis who have PTSD or symptoms of trauma; 2) to establish whether any one intervention is more effective; and 3) to outline implications for clinical practice and research. Outcomes of interests were identified a priori as follows: PTSD symptoms; quality of life; mental health symptoms; and adverse events.

\section{Method}

A protocol for the effectiveness of TFPT for psychosis and PTSD has previously been published in the Cochrane Library of Systematic Reviews (see Sin, Spain, Furuta, Murrells, \& Norman, 2015). The prior protocol served as a basis for this review, although the remit was broadened to include individuals experiencing trauma in the absence of a PTSD diagnosis. The review process followed PRISMA guidelines (Moher, Liberati, Tetzlaff, \& Altman, 2009). 


\section{Search strategy}

We searched the Cochrane Schizophrenia Group (CSG)'s study-based register of controlled trials (CENTRAL) - compiled from systematic searches of medical and social sciences databases (including AMED, EMBASE, MEDLINE, PsycINFO, and PubMed), clinical trials registers (including the EU, ISRCTN, WHO, and NIH registers) and sources of grey literature (including ProQuest theses and dissertations database) - using the following terms: (*trauma* or *ptsd*):ti,ab,kw of REFERENCE or (*trauma* or *ptsd*):sco of STUDY, from the date of inception until $28^{\text {th }}$ September 2015. Reference lists of included studies were also reviewed, and corresponding authors of studies screened were contacted for information regarding unpublished data and ongoing trials.

\section{Inclusion and exclusion criteria}

No language or publication sources limits were imposed. The inclusion criteria were as follows: 1) randomised controlled trials (RCTs); 2) investigating TFPT for PTSD, traumatic experiences, and/or the impact of these; 3 ) in adolescents or adults with a diagnosis of a non-organic psychotic disorder, including schizophrenia, psychosis, schizoaffective disorder and bipolar affective disorder (type 1); and 4) treated in any setting. Studies which recruited individuals diagnosed with a range of mental health disorders, a proportion of whom had psychosis, were included if either $50 \%$ of the sample had psychosis or when sub-group data were available. We excluded intervention studies where no specific outcome data pertaining to trauma were reported.

\section{Data Analysis}

Data were analysed using narrative and meta-analytic approaches (see Sin et al., 2015). Interventions were categorised into four main therapeutic approaches, as described by Bisson et al. (2013): individual TFCBT; group TFCBT; EMDR; and other psychological interventions not fitting into the above categories but which were clearly trauma-focused in their aims and remit. Separate analyses were undertaken to compare therapeutic approaches with inactive control conditions; when sufficient data were available, a head-tohead comparison was conducted between different interventions or active controls.

\section{Results}


Psychological interventions for comorbid trauma \& psychosis

\section{Study selection and search results}

Figure 1 outlines the search process and study selection. The search initially yielded 35 unique titles and abstracts. After examination, two duplicates were removed and two additional references were identified by contacting trial authors and reviewing trials registers. Eighteen references were excluded as their titles and abstracts were clearly irrelevant. Eight studies described in 17 references were assessed for eligibility. Three references were excluded following full-text examination, as one study did not employ an RCT design (de Bont, van Minnen, \& de Jongh, 2013); one did not provide trauma-related outcome data (Penn et al., 2011); and one related to an ongoing trial (Marlow, 2014; Marlow, 2015, personal communication).

\section{INSERT FIGURE 1: PRISMA flowchart HERE.}

\section{Overview of studies}

Five studies (outlined in 14 references), comprising 366 participants, met the review inclusion criteria (Jackson et al., 2009; Mueser et al., 2015; Mueser et al., 2008; Steel, 2010; van den Berg et al., 2015). See Table 1 for an overview of study details and participant characteristics. Studies were undertaken in the UK (Jackson et al., 2009; Steel, 2010), the Netherlands (van den Berg et al., 2015), and North America (Mueser et al., 2015; Mueser et al., 2008). Two studies investigated the effectiveness of TFCBT compared with usual care, using the same treatment protocol (Mueser et al., 2008; Steel 2010), one compared TFCBT with psychoeducation (Mueser et al., 2015), one investigated EMDR compared with prolonged exposure and a waitlist control (van den Berg et al., 2015), and one evaluated a cognitive therapy-based intervention (Cognitive Recovery Intervention, CRI) compared with usual care for people experiencing first episode psychosis (FEP) (Jackson et al., 2009).

\section{INSERT TABLE 1 HERE.}

\section{Quality assessment of studies}

Each study was independently assessed for risk of bias, specifically sequence generation, allocation concealment, blinding, incomplete outcome data and selective reporting, 
Psychological interventions for comorbid trauma \& psychosis

according to criteria described in the Cochrane Handbook for Systematic Reviews of Interventions (Higgins \& Green, 2011). See Figure 2. The trial designs and conduct were generally clearly reported, albeit that one study is as yet unpublished (Steel, 2015, personal communication). Of note, failure to recruit in one study ( $n=66$ ( $27 \%$ of 320 planned), Jackson et al., 2009) meant that trial analysis was significantly under-powered.

\section{INSERT FIGURE 2: Risk of bias summary for each included study HERE}

\section{Trauma experience: diagnosis, severity, and nature of symptoms}

Four studies included adults with a diagnosis of PTSD $(n=300)$ (Mueser et al., 2015;

Mueser et al., 2008; Steel, 2010; van den Berg et al., 2015), confirmed following assessment with the Clinician Administered PTSD Scale (CAPS by Blake et al., 1995). Jackson and colleagues (2009) used the Impact of Events Scale (IES by Sundin \& Horowitz, 2002) to measure post-traumatic phenomena in relation to FEP. Nonetheless, trial authors identified that 15 participants (23\% of the total sample) had a total IES score exceeding 40 points, strongly suggestive of a diagnosis of PTSD (Selley et al., 1997).

In two studies, it was reported that most participants had experienced multiple childhood traumas, including sexual, emotional and physical abuse (Mueser et al., 2008; van den Berg et al., 2015). van den Berg and colleagues also identified that 28 participants (18\% of the sample) developed PTSD as a consequence of traumatic psychosis experiences. Two studies did not describe the nature of traumatic events in detail (Mueser et al., 2015; Steel, 2010).

\section{Modalities of trauma-focused psychological interventions}

Across the studies, four trauma-focused interventions were delivered, on an individual basis. All interventions were manualised; therapists were provided with training and clinical supervision. Three studies (Mueser et al., 2008, Mueser et al., 2015; Steel, 2010) investigated the effectiveness of TFCBT based on cognitive models of PTSD (Ehlers, Clark, Hackmann, McManus, \& Fennell, 2005; Horowitz, Wilner, \& Alvarez, 1979). The intervention comprised psychoeducation about PTSD; breathing exercises; exposure-based sessions; and cognitive restructuring. Treatment was offered for 12-16 sessions, and participants were required to attend six or more sessions. One study (van den Berg et al., 2015) tested the effectiveness of an eight-session prolonged exposure therapy (PE), based upon an existing PTSD protocol (Foa, Hembree, \& Rothbaum, 2007), comprising case conceptualisation, and 
Psychological interventions for comorbid trauma \& psychosis

imaginal and in vivo exposure to a hierachy of trauma-related situations and cues. Jackson and colleagues (2009) investigated the utility of CRI, designed to enhance coping and adjustment following a FEP (which was deemed to be the index traumatic experience), and reduce the impact and distress associated with this. Participants were offered a maximum of 26 sessions, which involved three main facets: engagement and formulation; trauma processing; and appraisal of psychotic symptoms and experiences. An eight weekly $90-$ minute EMDR intervention, based on a Dutch translation of the standard EMDR protocol (de Jongh \& ten Broeke, 2003; Shapiro, 2001) was evaluated against TFCBT and waitlist control in the study based in the Netherlands (van den Berg et al., 2015). The intervention involved case formulation, identification of a hierarchy of traumatic experiences, and bilateral eye movements which were applied as the dual-attention stimuli to aid processing of traumatic memories. Lastly, one study (Mueser et al., 2015) offered a three session PTSD psychoeducation intervention, adapted from one used in a previous study about SMI and PTSD (Pratt et al., 2005), which included discussion about the causes and nature of PTSD, breathing exercises, and anxiety management.

\section{Effectiveness of interventions}

Statistical advice was sought regarding the appropriateness of undertaking meta-analyses (see Sin et al., 2015). Meta-analyses were undertaken using both random effects and fixedeffect models (using RevMan 5.3) to confirm the results were not significantly different regardless of models used. We presented the results analysed with the fixed-effect model here which was deemed to be more appropriate given the small number of studies (Higgins \& Green, 2011; Kontopantelis, Springate, \& Reeves, 2013). Based on the different intervention modalities, we examined the effectiveness of treatments using five comparisons: TFCBT versus usual care/waitlist; EMDR versus waitlist; EMDR versus TFCBT; PTSD psychoeducation versus TFCBT; and CRI versus usual care.

\section{TFCBT versus usual care/waitlist}

Three studies investigated the effectiveness of TFCBT $(n=160)$ (Mueser et al., 2008; Steel, 2010; van den Berg et al., 2015). Measures of treatment effect were calculated for several pre-specified outcomes, namely the CAPS (Blake et al., 1995; Weathers et al., 2001), and self-reported trauma-related cognitions (measured by Posttraumatic Cognitions Inventory (PTCl; Foa, Ehlers, Clark, Tolin, \& Orsillo, 1999). There was moderate quality evidence that TFCBT was associated with improved outcomes when compared with usual care or waitlist 
groups, in terms of reducing participants' clinician-rated PTSD symptoms (mean difference (MD) $-13.78,95 \%$ confidence interval $(\mathrm{Cl})=-23.67$ to -3.89 ) as well as self-reported traumarelated cognitions (MD $-19.46,95 \% \mathrm{Cl}=-35.05$ to -3.88 ) post-intervention, and at three to sixmonth follow up (see Figure 3a). Meta-analyses of pooled data from two studies $(n=113)$ (Mueser et al., 2008; van den Berg et al., 2015) provided some evidence that TFCBT was more effective in reducing PTSD symptoms to the extent that participants no longer met diagnostic criteria in the short and medium term (Risk Ratio $(\mathrm{RR}) 1.76,95 \% \mathrm{Cl}=1.13$ to 2.76 ) (see Figure 3b). As only one study provided data on outcomes of participants' self-reported PTSD symptoms and regarding full remission from PTSD (van den Berg, 2015), meta-analysis was precluded. Nonetheless, there is some limited evidence favouring TFCBT in these two outcomes, compared to a waitlist control.

Table 1 summarises the results of non-PTSD outcomes, specifically quality of life, wellbeing and mental health symptoms. It was not possible to pool the data as outcomes differed between studies. It was not clear whether TFCBT gleaned more favourable outcomes, compared with inactive controls.

One study provided data about rates of adverse events, and there were no differences between the two groups (van den Berg et al., 2015).

\section{INSERT FIGURES 3a - Forest plot of comparison: TFCBT versus usual care/waitlist on outcome of PTSD symptom severity as measured by CAPS (high = poor)} Figure 3b - Forest plot of comparison: TFCBT versus usual care/waitlist on outcome of loss of PTSD diagnosis (i.e. below diagnostic threshold as measured by CAPS)

\section{EMDR versus waitlist}

One study compared EMDR with TFCBT and a waitlist control (van den Berg et al., 2015). Meta-analysis was precluded. However, compared to the waitlist group $(n=102)$, EMDR was more effective in reducing clinician-rated (CAPS) and self-reported PTSD symptoms (PTCI and Posttraumatic Stress Symptom Scale Self-Report (PSS-SR) by Foa, Riggs, Dancu, \& Rothbaum, 1993). A statistically significant number of participants receiving EMDR attained sub-threshold PTSD symptoms (i.e. loss of PTSD diagnosis) post-treatment and at six-month follow up. There were no significant differences, between groups, in terms of rates of unspecified adverse events and loss to follow up. 
Psychological interventions for comorbid trauma \& psychosis

\section{EMDR versus TFCBT}

When comparing EMDR with TFCBT $(n=108)$, participants in both groups derived comparable benefits in self-rated and clinician-administered PTSD outcome measures (van den Berg et al., 2015).

\section{PTSD psychoeducation versus TFCBT}

One trial ( $n=67)$ compared TFCBT with PTSD psychoeducation (Mueser et al., 2015). Analyses of subgroup data did not provide evidence that this brief intervention gleaned greater improvement, compared with TFCBT across on CAPS and PTCI. There were no significant differences between groups in measures of quality of life, psychotic, and affective symptoms.

\section{CRI versus usual care}

One study evaluated the effectiveness of CRI compared to usual care, for reducing trauma, depression and low self-esteem in young adults following a FEP $(n=66)$ (Jackson et al., 2009). Participants who received CRI tended to have lower levels of post-intervention trauma symptoms, a finding which remained at six month follow up, particularly for those individuals who had high pre-treatment levels of trauma. Depression and self-esteem scores, however, were not significantly improved following the active intervention.

\section{Discussion}

Individuals who have psychosis often experience trauma and PTSD. This review summarised the effectiveness of TFPT for this comorbid population. Five studies met the pre-specified inclusion criteria. Overall, the review findings provide some good quality, albeit limited, evidence to support the use of TFPT, particularly those derived from cognitivebehavioural frameworks (TFCBT and CRI), and EMDR: active interventions were associated with improvement in clinician-rated and self-reported trauma symptoms. Benefits of interventions for low mood, anxiety and self-esteem, were equivocal. These findings are consistent with existing reviews about TFPT for non-psychosis population (e.g. Bisson et al., 
Psychological interventions for comorbid trauma \& psychosis

2013; Bradley et al., 2005); but additionally, provide preliminary support for interventions designed to address the experience, distress and impact of having psychosis.

Several factors potentially affect the generalisability of study findings. Although participants were recruited from clinical services, fairly stringent exclusion criteria were in place, including no recent inpatient admissions, changes to medication regime, and no coexisting substance dependence. The degree to which these criteria render a significant proportion of service users ineligible, is questionable. All bar one study (van den Berg et al., 2015) modified standard TFPT protocols so as to accommodate psychotic symptoms and associated cognitive processing difficulties (see Implications for practice below). Service constraints (for example, resources and staffing) and clinical complexity may imply that it is difficult to follow a protocol-derived treatment in routine care, as well as offering an extended course of sessions. Furthermore, as study participants also received usual care during the trial, this highlights the importance of continuous multi-disciplinary team input to address the often complex clinical needs and presentation.

\section{Limitations}

This review has several limitations. While the search strategy was rigorous and comprehensive, it is possible that studies which included a small proportion of individuals with psychosis may not have been retrieved, e.g. because trial authors subsumed psychosis under the umbrella term of SMI. Also, despite delineating between different intervention modalities, and analysing the data separately, there was some unexplained heterogeneity evident when comparing TFCBT with usual care. Consequently, the quality of evidence in several analyses was downgraded.

\section{Implications for practice}

There are several implications for clinical practice. Although assessment and intervention for psychotic symptoms often takes precedence, clinicians should be aware of the possibility that service users may have concurrent PTSD, or may previously or currently be experiencing trauma. Hence, assessment of PTSD symptoms is pragmatic when working with this clinical population, but decisions about when and how to do so, relies on individual need. This is particularly the case when working with people who have florid symptoms and high levels of distress. Assessment is likely to be enhanced if there is a strong therapeutic 
Psychological interventions for comorbid trauma \& psychosis

relationship between the clinician and service user, and may be informed by self-report questionnaires (e.g. PTCI, PSS-SR).

In terms of treatment, TFCBT and EMDR both appear to have clinical utility, augmented by concurrent MDT input. Study findings indicate that modifications are needed, in order to accommodate the unique needs of individuals with psychosis. Service users likely benefit from an extended course of treatment, with emphasis on engagement, development of therapeutic trust so that service users feel at ease and secure when discussing highly distressing and emotive topics, and additional relapse-prevention sessions. The duration of sessions should be based on individual need, i.e. depending on whether service users are able to sustain attention for an hour, or whether shorter sessions are better tolerated. Use of written and visual materials, that are simply laid out, concise, and focused, may be important for accommodating cognitive processing difficulties (Mueser et al., 2015; Mueser et al., 2008). To avoid overwhelming service users and to promote sequential hypothesis testing, it is important for clinicians to decide when to target PTSD symptoms, i.e. before or after other presenting difficulties. In general terms, clinicians should strive to integrate traumatic experiences and associated distress within the treatment formulation, in order to inform goals. This is crucial because firstly, traumatic experiences are likely to influence, and be influenced by, the individual's symptom presentation, but also as this may indirectly encourage concern or ambivalence about engaging with clinicians (Mueser et al., 2009; Read, Perry, Moskowitz, \& Connolly, 2001).

\section{Implications for research}

Building on the existing studies, we suggest further research endeavours are needed and could include: RCTs that compare different treatment modalities; consideration as to the optimal dose, i.e. number of sessions required to maximise treatment gains; assessment of acceptability and satisfaction with interventions; investigation into factors that mediate compliance, as well as treatment response (or lack thereof); and validation of PTSD self-report measures for individuals with psychosis (de Bont et al., 2015). Future research should also focus on establishing how best to provide therapists with training, so as to facilitate largerscale implementation of trauma-focused psychological interventions.

\section{Conclusion}


Psychological interventions for comorbid trauma \& psychosis

The findings of this review provides preliminary support for the use of TFPT, specifically TFCBT and EMDR for adults who have psychosis. Study results are comparable to nonpsychosis samples. The clinical implication is clear: assessment and treatment for PTSD and trauma symptoms are necessary in routine practice. Further research is needed to establish 1) which intervention modalities glean more favourable outcomes; 2) the optimum number of sessions required; and 3) how best to ensure that interventions are acceptable for service users. 
Psychological interventions for comorbid trauma \& psychosis

\section{References}

ACPMH. (2013). The Australian Guidelines for the Treatment for Adults with Acute Stress Disorder and Posttraumatic Stress Disorder. Melbourne: Australian Centre for Posttraumatic Mental Health. APA. (2013). Diagnostic and Statistical Manual of Mental Disorders. Arlington, VA: American Psychiatric Association.

Bebbington, P., Jonas, S., Kuipers, E., King, M., Cooper, C., Brugha, T., . . . Jenkins, R. (2011). Childhood sexual abuse and psychosis: data from a cross-sectional national psychiatric survey in England. British Journal of Psychiatry, 199, 19-37.

Bebbington, P. E., Bhugra, D., Brugha, T., Singleton, N., Farrell, M., Jenkins, R., .... Meltzer, H. (2004). Psychosis, victimisation and childhood disadvantage: evidence from the second British National Survey of Psychiatric Morbidity. British Journal of Psychiatry, 185, 220-226.

Birchwood, M. (2003). Pathways to emotional dysfunction in first episode psychosis. British Journal of Psychiatry, 182, 373-375.

Bisson, J. I., Roberts, N. P., Andrew, M., Cooper, R., \& Lewis, C. (2013). Psychological therapies for chronic post-traumatic stress disorder (PTSD) in adults. Cochrane Database of Systematic Reviews 2013, Issue 12. Art. No.: CD003388. DOI: 10/1002/14651858.CD003388.pub.4.

Blake, D. D., Weathers, F. W., Nagy, L. M., Kaloupek, D. G., Gusman, F. D., Charney, D. S., \& Keane, T. M. (1995). The development of a clinician administered PTSD scale. Journal of Traumatic Stress, 8(1), 75-90.

Bradley, R., Greene, J., Russ, E., Dutra, L., \& Westen, D. (2005). A multidimensional meta-analysis of psychotherapy for PTSD. The American Journal of Psychiatry, 162(2), 214-227.

Brunet, K., Birchwood, M., Upthegrove, R., Michail, M., \& Ross, K. (2012). A prospective study of PTSD following recovery from first-episode psychosis: the threat from persecutors, voices, and patienthood. British Journal of Clinical Psychology, 51, 418-433.

Callcott, P., Standart, S., \& Turkington, D. (2004). Trauma within psychosis: using a CBT model for PTSD in psychosis. Behavioural and Cognitive Psychotherapy, 32(2), 239-244.

Calvert, C., Larkin, W., \& Jellicoe-Jones, L. (2008). An exploration of the links between trauma and delusional ideation in secure services. Behavioural and Cognitive Psychotherapy, 36(5), 589-604.

Cunningham, T., Hoy, K., \& Shannon, C. (2015). Does childhood bullying lead to the development of psychotic symptoms? A meta-analysis and review of prospective studies. Psychosis. doi:

\subsection{0/17522439.2015.1053969}

Darves-Bornoz, J. M., Alonzo, J., de Girolamo, G., de Graaf, R., Haro, J. M., Kovess-Masfety, V., . . . On behalf of the ESEMeD/MHEDEA 2000 Investigators. (2008). Main traumatic events in Europe: PTSD in the European study of the epidemiology of mental disorders survey. Journal of Traumatic Stress, 21(5), 455-462.

de Bont, P. A., van Minnen, A., \& de Jongh, A. (2013). Treating PTSD in patients with psychosis: a within-group controlled feasibility study examining the efficacy and safety of evidence-based PE and EMDR protocols Behavior Therapy, 44, 717-730.

de Bont, P. A. J. M., van den Berg, D. P. G., van der Vleugel, B. M., de Roos, C., de Jongh, A., van der Gaag, M., \& van Minnen, A. (2015). Predictive validity of the Trauma Screening Questionnaire in detecting post-traumatic stress disorder in patients with psychotic disorders. British Journal of Psychiatry, 206, 408-416.

de Jongh, A., \& ten Broeke, E. (2003). Handbook of EMDR: A Standardised Treatent for the Consequences of Psychotrauma. Amsterdam, the Netherlands: Harcourt.

Ehlers, A., Clark, D. M., Hackmann, A., McManus, F., \& Fennell, M. (2005). Cognitive therapy for post-traumatic stress disorder: development and evaluation. Behaviour Research andTherapy, 43(4), 413-431.

Fisher, H. L., Schreier, A., Zammit, S., Maughan, B., Munafo, M. R., Lewis, G., \& Wolke, D. (2013). Pathways between childhood victimisation and psychosis-like symptoms in the ALSPAC birth cohort. Schizophrenia Bulletin, 39(5), 1045-1055.

Foa, E. B., Ehlers, A., Clark, D. M., Tolin, D. F., \& Orsillo, S. M. (1999). The Posttraumatic Cognitions Inventory (PTCl): Development and validation. Psychological Assessment, 11, 303-314. 
Foa, E. B., Hembree, E. A., \& Rothbaum, B. O. (2007). Prolonged Exposure Therapy for PTSD: Emotional Processing of Traumatic Experiences - Therapist Guide. Oxford, England: Oxford University Press.

Foa, E. B., Riggs, D. S., Dancu, C. V., \& Rothbaum, B. O. (1993). Reliability and validity of a brief instrument for assessing post-traumatic stress disorder. Journal of Traumatic Stress, 6, 459-473. Gairns, S., Alvarez-Jimenez, M., Hulbert, C., McGorry, P., \& Bendall, S. (2015). Perceptions of clinicians treating young people with first-episode psychosis for post-traumatic stress disorder. Early Intervention in Psychiatry, 9, 12-20.

Garety, P. A., Fowler, D. G., Freeman, D., Bebbington, P., Dunn, G., \& Kuipers, E. (2008). Cognitive behavioural therapy and family intervention for relapse prevention and symptom reduction in psychosis: randomised controlled trial. British Journal of Psychiatry, 192, 412-423.

Higgins, J. P. T., \& Green, S. (2011). Cochrane Handbook for Systematic Reviews of Interventions Version 5.0.2 [updated March 2011]. The Cochrane Collaboration, 2011 Available from www.cochrane-handbook.org.

Horowitz, M. J., Wilner, N. R., \& Alvarez, W. (1979). Impact of event scale - a measure of subjective stress. Psychosomatic Medicine, 41, 209-218.

Jackson, C., Trower, P., Reid, I., Smith, J., Hall, M., Townend, M., . . Birchwood, M. (2009). Improving psychological adjustment following a first episode of psychosis: a randomised controlled trial of cognitive therapy to reduce post psychotic trauma symptoms. Behaviour Research and Therapy, 47, 454-462.

Jones, V., \& Steel, C. (2014). Examining vulnerability to involuntary memories in schizophrenia comorbid with post-traumatic stress disorder. Schizophrenia Research, 152(2), 487-489.

Kessler, R. C., Sonnega, A., Bromet, E., Hughes, M., \& Nelson, C. B. (1995). Posttraumatic stress disorder in the National Comorbidity Survey. Archives of General Psychiatry, 52(12), 1048-1060. Kilcommons, A. M., \& Morrison, A. P. (2005). Relationships between trauma and psychosis: an exploration of cognitive and dissociative factors. Acta Psychiatrica Scandinavica, 112, 351-359. Kontopantelis, E., Springate, D. A., \& Reeves, D. (2013). A re-analysis of the Cochrane Library data: The dangers of unobserved heterogeneity in meta-analyses. PLOS ONE, 8(7), e69930-e69930. Marlow, S. (2014). Trauma Therapy for Psychosis: Eye Movement Desensitisation and Reprocessing (EMDR) for people with a psychotic illness. http://www.ISRCTN.com/ISRCTN43816889.

Moher, D., Liberati, A., Tetzlaff, J., \& Altman, D. G. (2009). Preferred Reporting Items for Systematic Reviews and Meta-Analysis: The PRISMA statement. BMJ, 339, b2535.

Morrison, A. P., Frame, L., \& Larkin, W. (2003). Relationships between trauma and psychosis: a review and integration. British Journal of Clinical Psychology, 42, 331-353.

Mueser, K. M., Rosenberg, S. D., \& Rosenberg, H. J. (2009). Treatment of Posttraumatic Stress Disorder in Special Populations: A Cognitive Restructuring Program. New York: American Psychological Association.

Mueser, K. T., Gottlieb, J. D., Xie, H., Lu, W., Yanos, P. T., Rosenberg, S. D., . . Wolfe, R. S. (2015). Evaluation of cognitive restructuring for post-traumatic stress disorder in people with severe mental illness. British Journal of Psychiatry, 206, 501-508.

Mueser, K. T., Lu, W., Rosenberg, S. D., \& Wolfe, R. (2010). The trauma of psychosis: posttraumatic stress disorder and recent onset psychosis. Schizophrenia Research, 116, 217-227.

Mueser, K. T., Rosenberg, S. D., Xie, H., Jankowski, M. K., Bolton, E. E., Lu, W., . . Wolfe, R. (2008). A randomized controlled trial of cognitive-behavioral treatment for posttraumatic stress disorder in severe mental illness Journal of Consulting and Clinical Psychology, 76, 259-271. NICE. (2005). Post-traumatic stress disorder (PTSD) - The management of PTSD in adults and children in primary and secondary care. London: National Institute of Clinical Excellence.

NICE. (2013). Post-traumatic stress disorder (PTSD) - Evidence Update December 2013 (Evidence Update 49). London: NICE.

NICE. (2014). Psychosis and Schizophrenia in Adults - Treatment and Mnagament (Clinical Guideline 136). London: National Collaborating Centre for Mental Health.

Penn, D., Uzeroff, S. R., Perkins, D., Mueser, K. T., R., H., Waldheter, E., . . Cook, L. (2011). A pilot investigation of the Graduated Recovery Intervention Program (GRIP) for First Episode Psychosis. Schizophrenia Research, 125(2-3), 247-256. 
Pratt, S. I., Rosenberg, S. D., Mueser, K. T., Brancato, J., Salyers, M. P., Jankowski, M. K., \& Descamp, S. M. (2005). Evaluation of a PTSD psychoeducational program for psychiatric inpatients. Journal of Mental Health, 14, 121-127.

Read, J., Fink, P. J., Rudegeair, T., Felitti, V., \& Whitfield, C. L. (2008). Child maltreatment and psychosis: a return to a genuinely integrated bio-psycho-social model. Clinical Schizophrenia \& Related Psychoses, 2(3), 235-254.

Read, J., Perry, B. D., Moskowitz, A., \& Connolly, J. (2001). The contribution of early traumatic events to schizophrenia in some patients: a traumagenic developmental model. Psychiatry, 64(4), 319-345.

Schnyder, U., Ehlers, A., Elbert, T., Foa, E. B., Gersons, B. P. R., Resick, P. A., . . Cloitre, M. (2015). Psychotherapies fpr PTSD: what do they have in common? European Journal of Psychotraumatology, 6, 28186.

Selley, C., King, E., Peveler, R., Osola, K., Martin, N., \& Thompson, C. (1997). Post-traumatic stress disorder symptoms and the Clapham rail accident. British Journal of Psychiatry, 171, 478-482.

Shapiro, F. (2001). Eye Movement Desensitization and Reprocessing, Basic Principles, Protocols and Procesures (2nd Edition). New York: The Guilford Press.

Sin, J., Spain, D., Furuta, M., Murrells, T., \& Norman, I. (2015). Psychological interventons for posttraumatic stress disorder (PTSD) in people with severe mental illness. Cochrane Database of Systematic Reviews 2013, Issue 1. Art. No.: CD011464. DOI: 10.1002/14651858. CD011464. Steel, C. (2010). The acceptability and effectiveness of cognitive behavioural therapy for the treatment of post-traumatic stress disorder within schizophrenia http://www.controlled-trials.com. Sundin, E. C., \& Horowitz, M. J. (2002). Impact of Event Scale: psychometric properties. British Journal of Psychiatry, 180, 2205-2209.

The Schizophrenia Commission. (2012). The abandoned illness: a report from the Schizophrenia Commission. London: Rethink Mental Illness.

Turkington, D., Kingdon, D., \& Turner, T. (2002). Effectiveness of a brief cognitive behavioural therapy intervention in the treatment of schizophrenia. British Journal of Psychiatry, 180, 523-527. van den Berg, D. P. G., de Bont, P. A. J. M., van der Vieugel, B. M., de Roos, C., de Jongh, A., Van Minnen, A., \& van der Gaag, M. (2015). Prolonged exposure vs Eye Movement Desensitization and Reprocessing vs waiting list for posttraumatic stress disorder in patients with a psychotic disorder - A randomised clinical trial. JAMA Psychiatry. DOI: 10.1001/jamapsychiatry.2014.2637.

Varese, F., Smeets, F., Drukker, M., Lieverse, R., Lataster, T., Viechtbauer, W., . . Bentall, R. P. (2012). Childhood adversities increase the risk of psychosis: a meta-analysis of patient-control, prospective- and cross-sectional cohort studies. Schizophrenia Bulletin, 38(4), 661-671.

Weathers, F. W., Keane, T. M., \& Davidson, J. R. (2001). Clinician-Administered PTSD Scale: a review of the first ten years of research. Depression and Anxiety, 13(3), 132-156. 
Table 1: Summary of included studies

\begin{tabular}{|c|c|c|c|c|}
\hline Study & $\begin{array}{l}\text { Participants \& } \\
\text { conditions }\end{array}$ & Intervention & Outcomes & Results \\
\hline $\begin{array}{l}\text { Jackson et al., } \\
\text { 2009; Jackson et } \\
\text { al., 2004; Jackson } \\
\text { et al., 2006; } \\
\text { Jackson 2001; } \\
\text { Jackson 2004; } \\
\text { Jackson, } 2003 \\
\text { United Kingdom }\end{array}$ & $\begin{array}{l}66 \text { participants }(74 \% \\
\text { male, mean age }=23.3, \\
S D=4.6) \text { aged between } \\
16 \text { and } 35 \text { with FEP } \\
N=36 \text { in Cognitive } \\
\text { Recovery Intervention } \\
(\mathrm{CRI}) \\
\mathrm{N}=30 \text { in TAU } \\
\text { Ethnicity: } 73 \% \text { White; } 5 \% \\
\text { Black; } 15 \% \text { South Asian; } \\
7 \% \text { Mixed race }\end{array}$ & $\begin{array}{l}\text { - Individual CRI designed to support } \\
\text { service users adjusting to and } \\
\text { recovering from a FEP. } \\
\text { - A maximum of } 26 \text { sessions delivered } \\
\text { on a weekly basis over } 6 \text { months. } \\
\text { - CRI has three key components: (a) } \\
\text { engagement and formulation; (b) } \\
\text { trauma processing; and (c) appraisals } \\
\text { of psychotic illness, and works towards } \\
\text { an "individual recovery plan". }\end{array}$ & $\begin{array}{l}\text { - Trauma symptoms: IES } \\
\text { - Depression: CDSS } \\
\text { - Self-esteem: SCQ }\end{array}$ & $\begin{array}{l}\text { - CRI participants fared } \\
\text { marginally better on IES than } \\
\text { those in the control group; this } \\
\text { was especially the case at } 6 \\
\text { months for those with high pre- } \\
\text { treatment levels of trauma } \\
\text { symptoms. } \\
\text { - No significant difference } \\
\text { identified groups on CDSS and } \\
\text { SCQ scores. } \\
\text { - Total attrition across groups = } \\
32 \% \text { at } 12 \text { month follow up; } \\
\text { similar rates of attrition } \\
\text { between groups. }\end{array}$ \\
\hline $\begin{array}{l}\text { Mueser et al., } \\
\text { 2008; Mueser et } \\
\text { al., 2003; Mueser } \\
\text { et al., } 2015 \\
\text { unpublished } \\
\text { subgroup data } \\
\text { United States }\end{array}$ & $\begin{array}{l}17^{\star} \text { adults (no } \\
\text { demographic data on } \\
\text { subgroup sample) with } \\
\text { comorbid PTSD and } \\
\text { schizophrenia or } \\
\text { schizoaffective disorder } \\
\text { among the total number } \\
\text { of participants (N = } \\
108^{\star *}, 21 \% \text { male, mean } \\
\text { age }=44.2, \mathrm{SD}=10.6 \text { ) } \\
\text { with comorbid PTSD } \\
\text { and various SMI } \\
\text { diagnoses } \\
\mathrm{N}=10 \text { in TFCBT + TAU } \\
\mathrm{N}=7 \text { in TAU } \\
\text { Ethnicity**: } 84 \% \text { White; } \\
16 \% \text { other }\end{array}$ & $\begin{array}{l}\text { - TFCBT, an individual intervention } \\
\text { specifically designed for the comorbid } \\
\text { population } \\
\text { - A programme of } 12 \text { to } 16 \text { sessions } \\
\text { delivered over } 6 \text { months. } \\
\text { - TFCBT used a structured format } \\
\text { inclusive of handouts, worksheets, } \\
\text { and homework assignments; covering } \\
\text { key contents of: treatment overview, } \\
\text { psychoeducation, breathing retraining } \\
\text { and cognitive restructuring as from the } \\
\text { sixth sessions. }\end{array}$ & $\begin{array}{l}\text { - PTSD symptom severity: CAPS } \\
\text { - Trauma-related cognitions: PTCI } \\
\text { - Loss of PTSD diagnosis }{ }^{1}: \text { CAPS } \\
\text { cut off score } \\
\text { - Mental health functioning: SF-12 } \\
\text { mental component } \\
\text { - Physical functioning: SF-12 } \\
\text { physical component } \\
\text { - Overall psychiatric symptoms: } \\
\text { BPRS } \\
\text { - Depression: BDI-II } \\
\text { - Anxiety: BAI }\end{array}$ & $\begin{array}{l}\text { - No significant difference } \\
\text { identified from the psychosis } \\
\text { subgroup data across the two } \\
\text { arms in all the specified } \\
\text { outcomes. } \\
\text { - Total attrition }=47 \% \text { at } 12 \\
\text { month follow up; loss to follow } \\
\text { up was similar between } \\
\text { groups. }\end{array}$ \\
\hline
\end{tabular}




\begin{tabular}{|c|c|c|c|c|}
\hline Study & $\begin{array}{l}\text { Participants \& } \\
\text { conditions }\end{array}$ & Intervention & Outcomes & Results \\
\hline $\begin{array}{l}\text { Mueser et al., } \\
\text { 2015; Mueser et } \\
\text { al., 2007; Mueser } \\
\text { et al., } 2015 \\
\text { unpublished } \\
\text { subgroup data } \\
\text { United States }\end{array}$ & $\begin{array}{l}67^{\star} \text { adults }(39 \% \text { male, } \\
\text { mean age = } 43.4, \text { SD }= \\
12.0 \text { ) with comorbid } \\
\text { severe PTSD and } \\
\text { schizophrenia or } \\
\text { schizoaffective disorder } \\
\text { among the total number } \\
\text { of participants }(\mathrm{N}= \\
201)^{\star \star} \text { with comorbid } \\
\text { PTSD and various SMI } \\
\text { diagnoses } \\
\mathrm{N}=32 \text { in TFCBT + TAU } \\
\mathrm{N}=35 \text { in Brief PTSD } \\
\text { Psychoeducation } \\
\text { Programme (BPPP) + } \\
\text { TAU } \\
\text { Ethnicity: } 21 \% \text { White; } \\
\text { 15\% Hispanic; } 64 \% \text { non- } \\
\text { White }\end{array}$ & $\begin{array}{l}\text { - Individual-based TFCBT specially } \\
\text { designed and adapted for participants } \\
\text { with psychosis } \\
\text { - A programme of } 12 \text { - to 16- sessions } \\
\text { delivered over } 6 \text { months. } \\
\text { - TFCBT programme included } 3 \\
\text { sessions teaching breathing for } \\
\text { anxiety and education about trauma } \\
\text { and PTSD, followed by } 9-13 \text { sessions } \\
\text { of cognitive restructuring. } \\
\text { - BPPP was an individual treatment } \\
\text { focusing on educating people with SMI } \\
\text { on PTSD and related symptoms- } \\
\text { management } \\
\text { - } 3 \text { sessions run over a month. } \\
\text { - Content covered the first } 3 \text { sessions of } \\
\text { TFCBT programme, no cognitive } \\
\text { restructuring. }\end{array}$ & $\begin{array}{l}\text { - PTSD symptom severity: CAPS } \\
\text { - Trauma-related cognitions: PTCI } \\
\text { - Loss of PTSD diagnosis: }{ }^{1} \text { CAPS } \\
\text { cut off score } \\
\text { - Loss of severe PTSD diagnosis: } \\
\text { CAPS score< } 65 \\
\text { - Quality of life across different life } \\
\text { domains: QoLI } \\
\text { - Overall functioning: Global: GAF } \\
\text { - Social functioning: CAPS-social } \\
\text { functioning sub-scale } \\
\text { - Psychiatric symptoms: PANSS } \\
\text { - Depression: BDI-II } \\
\text { - Anxiety: BAI }\end{array}$ & $\begin{array}{l}\text { - No significant difference } \\
\text { identified in the psychosis } \\
\text { subgroup data across the } \\
\text { TFCBT and BPPP arms in all } \\
\text { specified outcomes. } \\
\text { - Total attrition }=28 \% \text { at } 18 \\
\text { month follow up; no significant } \\
\text { difference in attrition rates } \\
\text { between groups. }\end{array}$ \\
\hline $\begin{array}{l}\text { Steel, } 2010 ; \\
\text { Steel, } \quad 2015 \\
\text { unpublished } \\
\text { data } \\
\text { United Kingdom }\end{array}$ & $\begin{array}{l}61 \text { adults with } \\
\text { schizophrenia or } \\
\text { schizoaffective disorder } \\
\text { and concurrent PTSD } \\
N=30 \text { in TFCBT + TAU } \\
N=31 \text { in TAU } \\
\text { No available data on } \\
\text { age, gender or ethnicity } \\
\text { of participants. }\end{array}$ & $\begin{array}{l}\text { - This study adopted the16-session } \\
\text { TFCBT developed by Mueser et al., } \\
\underline{2008} \text { (see above for further details) }\end{array}$ & $\begin{array}{l}\text { - PTSD symptom severity: CAPS } \\
\text { - Posttraumatic cognitions: PTCI } \\
\text { - Quality of life: QOLS } \\
\text { - Overall functioning: GAF } \\
\text { - Positive psychotic } \\
\text { symptomatology: PANSS positive } \\
\text { subscale total } \\
\text { - Negative psychotic } \\
\text { symptomatology: PANSS negative } \\
\text { subscale total } \\
\text { - Auditory hallucinations: PSYRATS } \\
\text { hallucinations subscale total } \\
\text { - Delusions: PSYRATS delusions } \\
\text { subscale total }\end{array}$ & $\begin{array}{l}\text { - No significant difference } \\
\text { identified between the TFCBT } \\
\text { and TAU groups in all the } \\
\text { specified outcomes. } \\
\text { - Attrition across groups was } \\
\text { similar; total attrition rate = } \\
23 \% \text {. }\end{array}$ \\
\hline
\end{tabular}




\begin{tabular}{lllcl}
\hline Study & $\begin{array}{l}\text { Participants \& } \\
\text { conditions }\end{array}$ & Intervention & Outcomes & Results \\
\hline
\end{tabular}

- Depression: BDI-II

- Anxiety: BAl

van den Berg et 155 adults (46\% male,

al., 2015; de Bont mean age $=41.2, \mathrm{SD}=$

et al., 2013; de 10.5) with a psychotic

Bont et al., 2012

disorder or mood

disorder with psychotic

features and chronic

PTSD

$\mathrm{N}=53$ in Prolonged

Exposure (PE) + TAU

$\mathrm{N}=55$ in EMDR + TAU

$\mathrm{N}=47$ in Waitlist + TAU

Ethnicity: 63\% Dutch;

$31 \%$ non-Western; 6\%

Western, non-Dutch
- PE therapy was based on a protocol

by Foa et al., 2007.

- PE included 8 weekly 90-minute

sessions offered over 10 weeks.

- PE sessions comprised development

of a case formulation including a

hierarchy of trauma-related stimuli,

and then use of imaginal and in vivo

exposure; audio recordings of

sessions were made and listened to 5

times a week for homework.

- EMDR followed the Dutch protocol (de

Jongh \& ten Broeke, 2003) translated

from Shapiro (2001) manual

- EMDR was delivered over 8 weekly

90-minute sessions, in 10 weeks.

- EMDR group used the first session to

develop a case conceptualisation,

then traumatic memories were

processed from the second session

through to the eighth, with bilateral

eye movement applied.
- PTSD symptom: CAPS

- Self-reported frequency of PTSD

symptoms: PSS-SR

- Trauma-related cognitions: PTCI

- Loss of PTSD diagnosis ${ }^{1}$ : CAPS score $<40$

- Full remission from PTSD2: CAPS

score $<20$

- Severe adverse events

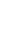

de




\begin{tabular}{|c|c|c|c|c|c|}
\hline Study & $\begin{array}{l}\text { Participants \& } \\
\text { conditions }\end{array}$ & Intervention & Outcomes & Results & \\
\hline & & & & $\begin{array}{l}\text { difference identified } \\
\text { groups. }\end{array}$ & across \\
\hline
\end{tabular}

Bold print denotes the paper as the primary reference for the study; *psychotic disorder subgroup data used in the review; **total original sample size and characteristics reported by the paper; ${ }^{1}$ Loss of PTSD diagnosis was defined by studies as PTSD symptoms no longer meeting DSM-IV diagnostic criteria as measured by CAPS cut off score of below 40; ${ }^{2}$ Full remission of PTSD was defined by studies as achieving CAPS score of below 20 (Weathers et al., 2001 )

IES - Impact of Events Scale; CDSS - Calgary Depression Scale; SCQ - Robson Self Esteem Questionnaire; CAPS - Clinician Administered PTSD; PANSS - Positive and Negative Syndrome Scale: QOLI - Brief Quality of Life interview; GAF - Global Assessment of Functioning Scale; PTCI - Posttraumatic

Cognitions Inventory; BDI- Beck Depression Inventory-II; BAI - Beck Anxiety Inventory; BPRS - expanded version of the Brief Psychiatric Rating Scale; SF-12 - 12 physical component; QOLS - Quality of Life Scale; PSYRATS - Psychotic Symptom Rating Scale; PSS-SR - Posttraumatic Stress Symptom Scale Self Report; TAU = treatment as usual. 
Figure 1: PRISMA flowchart

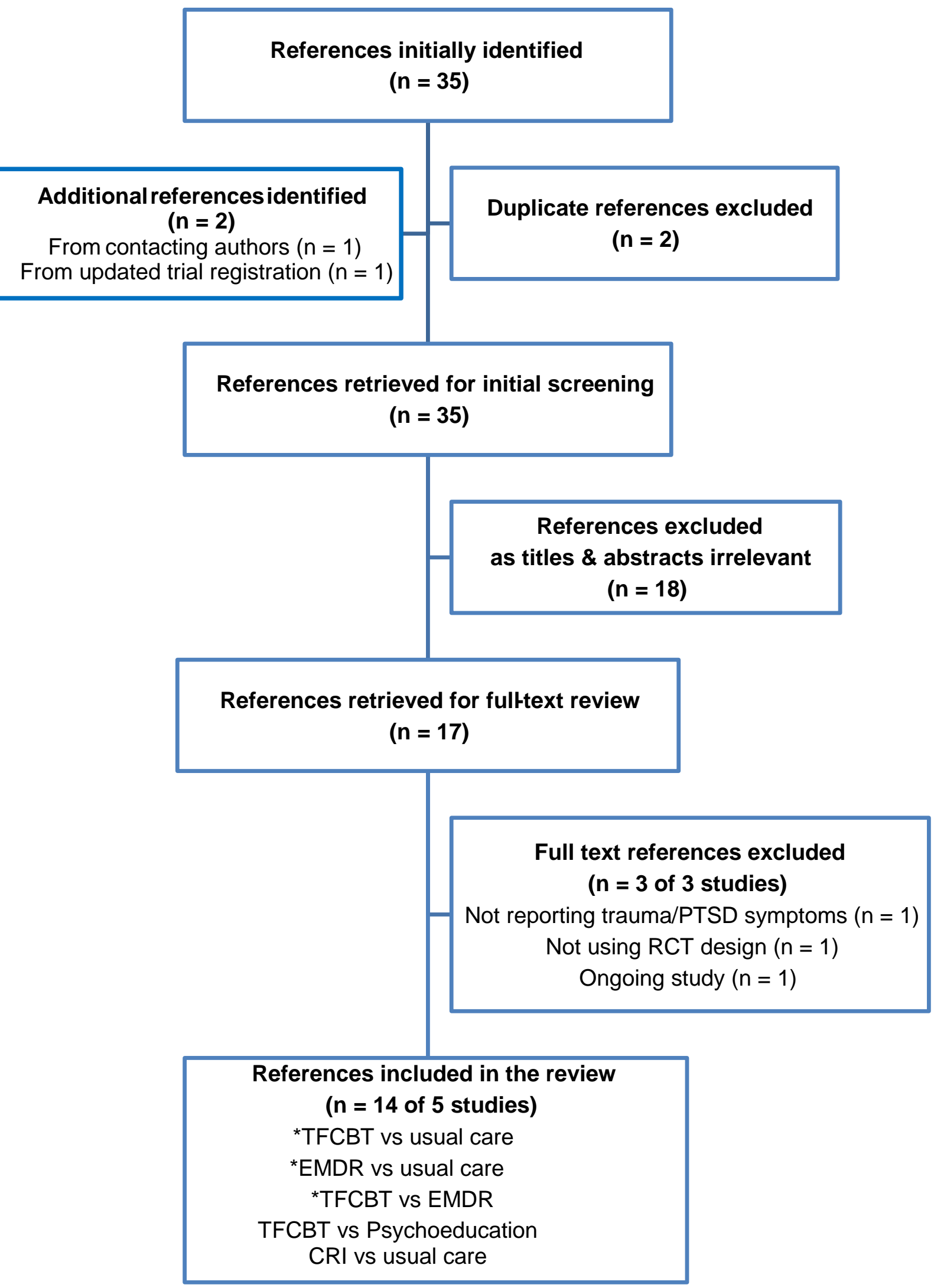

*One study (3 papers) reported a three-arm trial comparing TFCBT, EMDR and waitlist control. 
Psychological interventions for comorbid trauma \& psychosis

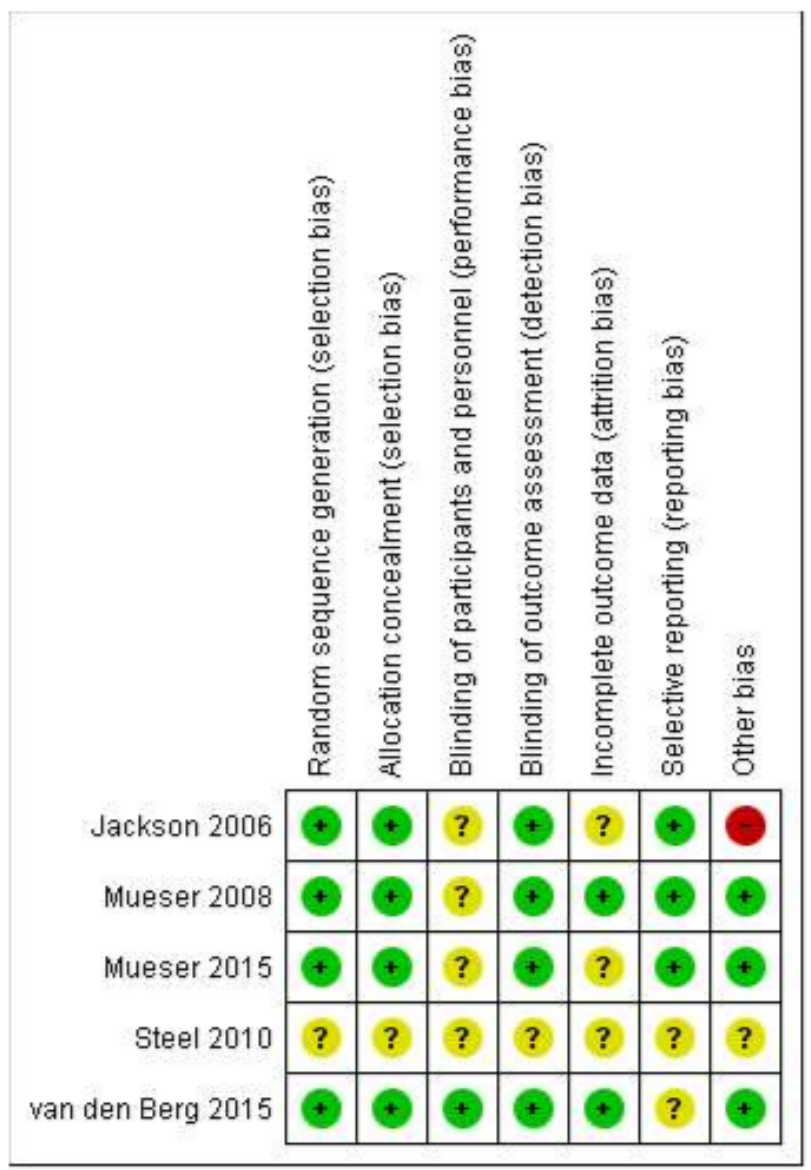

Figure 3a - Forest plot of comparison: Individual TFCBT versus usual care on outcome of PTSD symptom severity - as measured by CAPS (high = poor)

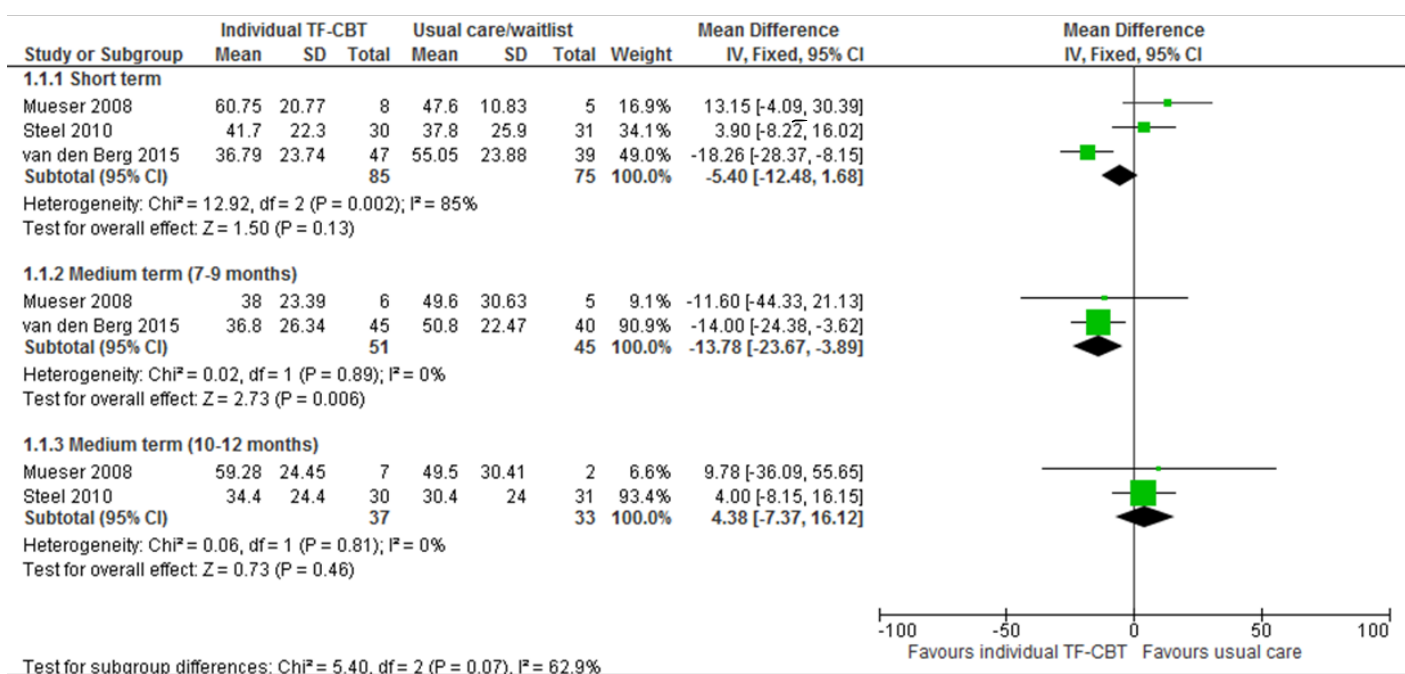


Figure 3b - Forest plot of comparison: Individual TFCBT versus usual care on outcome of loss of PTSD diagnosis (below diagnostic threshold as measured by CAPS)

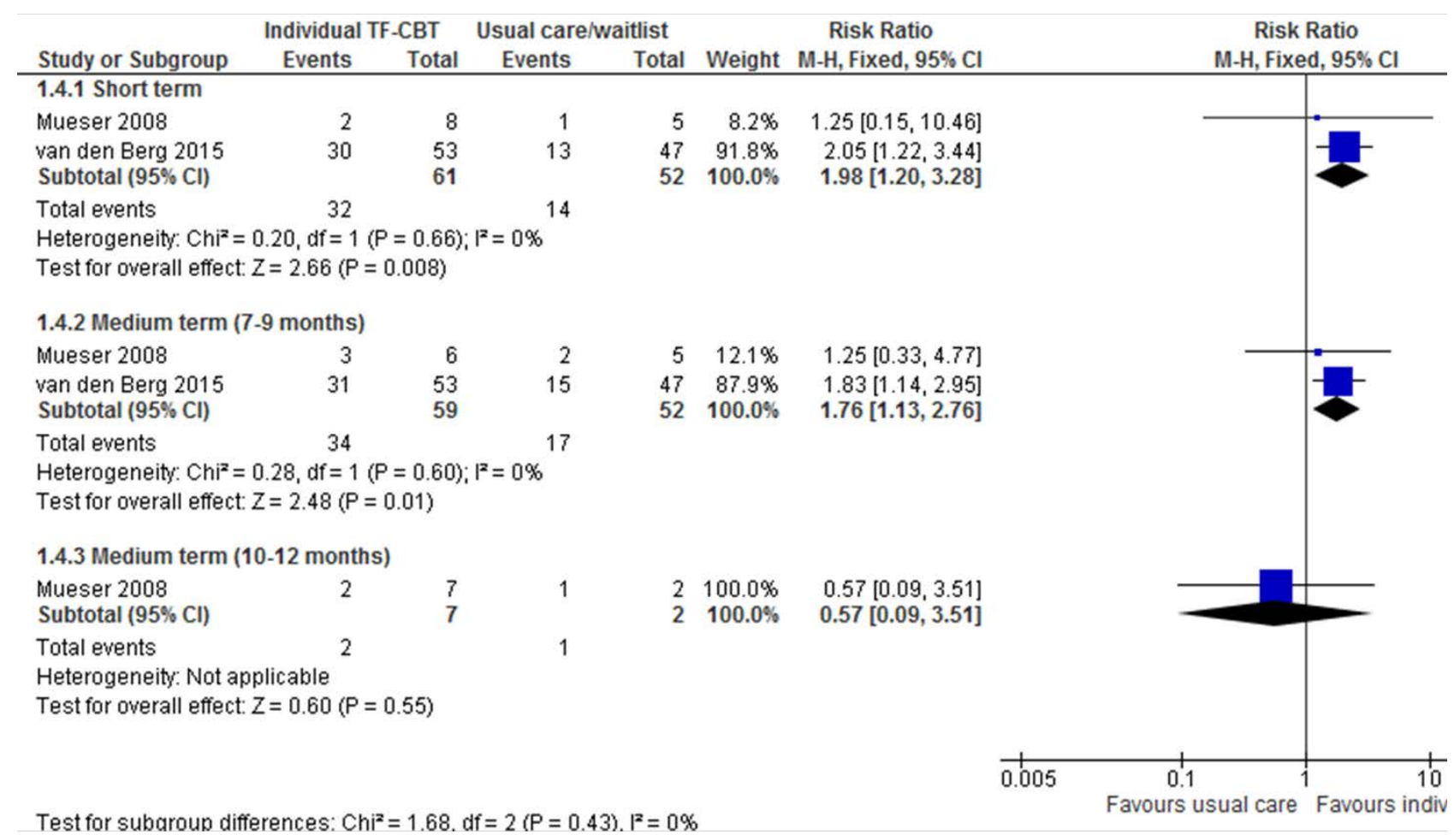

